\title{
Superconvergence of triangular mixed finite element methods for nonlinear optimal control problems
}

\author{
Zuliang Lu${ }^{\mathrm{a}, *}$, Shuhua Zhang ${ }^{\mathrm{b}}$ \\ a Key Laboratory for Nonlinear Science and System Structure, \\ Key Laboratory of Signal and Information Processing, Chongqing Three Gorges University, \\ Chongqing 404000, China \\ b Research Centre for Mathematics and Economics, Tianjin University of Finance and Economics, \\ Tianjin 300222, China \\ *Corresponding author, e-mail: zulianglux@126.com
}

Received 9 Apr 2015

Accepted 10 Apr 2016

\begin{abstract}
In this paper, we investigate the superconvergence of nonlinear elliptic optimal control problems by using triangular mixed finite element methods. The state and the co-state are approximated by the lowest order RaviartThomas mixed finite element spaces and the control is approximated by piecewise constant functions. We obtain the superconvergence of $O\left(h^{3 / 2}\right)$ for the control variable and coupled state variable. Numerical results demonstrating these superconvergence results are also presented.
\end{abstract}

KEYWORDS: lowest order Raviart-Thomas mixed finite element methods, triangular partition

MSC2010: 49J20 65N30

\section{INTRODUCTION}

Optimal control problems play increasingly important role in multi-disciplinary applications such as engineering design, fluid mechanics, physical, biological, medicine, finance, and social-economic systems. There are various numerical methods to solve these complex problems. Among these numerical methods, finite element methods for state equations have many applications. Papers devoted to linearquadratic optimal control problems include those by Falk ${ }^{1}$ and Geveci ${ }^{2}$. The authors studied the numerical approximation of distributed nonlinear optimal control problems with pointwise constraints on the control ${ }^{3}$. Meyer and Rösch ${ }^{4}$ analysed finite element discretization of the dimensional (2-d) elliptic optimal control problem. These approximations have convergence of order $h^{2}$. A posteriori error estimates for distributed convex optimal control problems and nonlinear optimal control problems have been obtained ${ }^{5,6}$.

Compared with standard finite element methods, mixed finite element methods have many advantages. In many control problems, the objective functional contains the gradient of the state variables. Thus the accuracy of the gradient is important in the numerical discretization of the coupled state equations. Mixed finite element methods are appropriate for the state equations in such cases since both the scalar variable and its flux variable can be approximated to the same accuracy by using such methods.

Recently, we obtained a priori error estimates and a posteriori error estimates of mixed finite element methods for linear and nonlinear optimal control problems ${ }^{7-9}$. Then we used the postprocessing projection operator to prove a quadratic superconvergence of the control for linear elliptic optimal control problem by a mixed finite element method $^{10-12}$.

We are concerned with the 2-d nonlinear elliptic optimal control problem

$$
\min _{u \in U_{\text {ad }}}\left\{\frac{1}{2}\left\|\mathbf{p}-\mathbf{p}_{\mathrm{d}}\right\|^{2}+\frac{1}{2}\left\|y-y_{\mathrm{d}}\right\|^{2}+\frac{\alpha}{2}\|u\|^{2}\right\}
$$

subject to the state equations

$$
\operatorname{div} \mathbf{p}+\phi(y)=u, \mathbf{p}=-A \nabla y, \quad x \in \Omega,
$$

with the boundary condition

$$
y=0, x \in \partial \Omega,
$$

where $\Omega$ is a rectangular domain, $\mathbf{p}_{\mathrm{d}}$ and $y_{\mathrm{d}}$ are two known functions, $\mathbf{p}$ and $y$ are state variables, $u$ is a 
control variable, and $v>0$ is a constant. We denote the set of admissible controls by $U_{\text {ad }}$, where

$$
U_{\mathrm{ad}}=\left\{u \in L^{2}(\Omega): u \geqslant 0 \text { a.e. in } \Omega\right\} .
$$

Let us state the assumptions on the operator $A$ and the functional $\phi$ : (A1) the coefficient matrix function $A(x)=\left(a_{i j}(x)\right)$ is symmetric with $a_{i j}(x) \in$ $W^{1, \infty}(\Omega)$, which satisfies the ellipticity condition $c_{*}|\xi|^{2} \leqslant \sum_{i, j=1}^{2} a_{i j}(x) \xi_{i} \xi_{j} \leqslant c^{*}|\xi|^{2}, \forall(\xi, x) \in \mathbb{R}^{2} \times \bar{\Omega}$, $c_{*}, c^{*}>0$; (A2) $\phi$ is of class $C^{2}$ with respect to the variable $y$, for any $R>0$ the function $\phi(\cdot) \in$ $W^{2, \infty}(-R, R), \phi^{\prime}(y) \in L^{2}(\Omega)$ for any $y \in H^{1}(\Omega)$, and $\phi^{\prime}(y) \geqslant \lambda>0$.

Lemma 1 (Ref. 13) For every function $g \in L^{p}(\Omega)$ $(p \geqslant 1)$, the solution $y$ of

$$
-\operatorname{div}(A \nabla y)+\phi(y)=g \text { in } \Omega,\left.y\right|_{\partial \Omega}=0,
$$

belongs to $H_{0}^{1}(\Omega) \cap W^{2, p}(\Omega)$. Moreover, there exists a positive constant $C$ such that

$$
\|y\|_{W^{2, p}(\Omega)} \leqslant C\|g\|_{L^{p}(\Omega)} .
$$

Next, we introduce the co-state elliptic equations

$$
\operatorname{div} \mathbf{q}+\phi^{\prime}(y) z=y-y_{\mathrm{d}}, \mathbf{q}=-A\left(\nabla z+\mathbf{p}-\mathbf{p}_{\mathrm{d}}\right),
$$

with boundary condition $z=0, x \in \partial \Omega$. The existence of a unique solution of (2) and (4) is justified by Lemma 1. Furthermore, we make the following realistic assumption (A3): $u \in W^{1, \infty}(\Omega)$, $y, z \in H^{3}(\Omega)$.

\section{MIXED METHODS FOR OPTIMAL CONTROL PROBLEM}

We shall construct a discretized scheme for the nonlinear optimal control problem (1)-(3) by using mixed finite element methods and give its equivalent optimality conditions.

$$
\text { Let } W=L^{2}(\Omega) \text {, }
$$

$$
\mathbf{V}=H(\operatorname{div} ; \Omega)=\left\{\mathbf{v} \in L^{2}(\Omega)^{2}, \operatorname{div} \mathbf{v} \in L^{2}(\Omega)\right\} .
$$

The Hilbert space $\mathbf{V}$ is equipped with the following norm: $\|\mathbf{v}\|_{\text {div }}=\|\mathbf{v}\|_{H(\operatorname{div} ; \Omega)}=\left(\|\mathbf{v}\|^{2}+\|\operatorname{div} \mathbf{v}\|^{2}\right)^{1 / 2}$. A weak formulation of the optimal control problem (1)-(3) is to find ( $\mathbf{p}, y, u) \in \mathbf{V} \times W \times U_{\text {ad }}$ such that

$$
\begin{gathered}
\min _{u \in U_{\text {ad }}}\left\{\frac{1}{2}\left\|\mathbf{p}-\mathbf{p}_{\mathrm{d}}\right\|^{2}+\frac{1}{2}\left\|y-y_{\mathrm{d}}\right\|^{2}+\frac{\alpha}{2}\|u\|^{2}\right\} \\
\left(A^{-1} \mathbf{p}, \mathbf{v}\right)-(y, \operatorname{div} \mathbf{v})=0, \quad \forall \mathbf{v} \in \mathbf{V}, \\
(\operatorname{div} \mathbf{p}, w)+(\phi(y), w)=(u, w), \quad \forall w \in W,
\end{gathered}
$$

where the inner product in $L^{2}(\Omega)$ or $\left(L^{2}(\Omega)\right)^{2}$ is denoted by $(\cdot, \cdot)$. It is well known ${ }^{14}$ that the convex control problem (5)-(7) has a solution $\left(\mathbf{p}^{*}, y^{*}, u^{*}\right)$, and that if a triplet $\left(\mathbf{p}^{*}, y^{*}, u^{*}\right) \in \mathbf{V} \times W \times U$ is the solution of (5)-(7), then there exists a co-state $\left(\mathbf{q}^{*}, z^{*}\right) \in \mathbf{V} \times W$ such that $\left(\mathbf{p}^{*}, y^{*}, \mathbf{q}^{*}, z^{*}, u^{*}\right)$ satisfies the following optimality conditions:

$$
\begin{gathered}
\left(A^{-1} \mathbf{p}^{*}, \mathbf{v}\right)-\left(y^{*}, \operatorname{div} \mathbf{v}\right)=0, \\
\left(\operatorname{div} \mathbf{p}^{*}, w\right)+\left(\phi\left(y^{*}\right), w\right)=\left(u^{*}, w\right), \\
\left(A^{-1} \mathbf{q}^{*}, \mathbf{v}\right)-\left(z^{*}, \operatorname{div} \mathbf{v}\right)=-\left(\mathbf{p}^{*}-\mathbf{p}_{\mathrm{d}}, \mathbf{v}\right), \\
\left(\operatorname{div} \mathbf{q}^{*}, w\right)+\left(\phi^{\prime}\left(y^{*}\right) z^{*}, w\right)=\left(y^{*}-y_{\mathrm{d}}, w\right), \\
\left(z^{*}+\alpha u^{*}, \tilde{u}-u^{*}\right) \geqslant 0,
\end{gathered}
$$

where $\mathbf{v} \in \mathbf{V}, w \in W$ and $\tilde{u} \in U_{\text {ad }}$.

We now introduce the discretized problem by considering a family of triangulations $\mathscr{T}_{h}$ of $\bar{\Omega}$. With each element $T_{i} \in \mathscr{T}_{h}$, we associate two parameters $\rho\left(T_{i}\right)$ and $\sigma\left(T_{i}\right)$, where $\rho\left(T_{i}\right)$ denotes the diameter of the set $T_{i}$ and $\sigma\left(T_{i}\right)$ is the diameter of the largest ball contained in $T_{i}$. The mesh size of the grid is defined by $h=\max _{T_{i} \in \mathscr{T}_{h}} \rho\left(T_{i}\right)$. We suppose that the following regularity assumptions are satisfied. There exist two positive constants $\varrho_{1}$ and $\varrho_{2}$ such that $\left(\rho\left(T_{i}\right) / \sigma\left(T_{i}\right)\right) \leqslant \varrho_{1},\left(h / \rho\left(T_{i}\right)\right) \leqslant \varrho_{2}$ hold for all $T_{i} \in \mathscr{T}_{h}$ and all $h>0$.

Let $\mathbf{V}_{h} \times W_{h} \subset \mathbf{V} \times W$ denote the lowest order Raviart-Thomas mixed finite element space ${ }^{15}$, namely,

$$
\begin{gathered}
\mathbf{V}_{h}=\left\{\mathbf{v} \in \mathbf{V}: \forall T_{i} \in \mathscr{T}_{h},\left.\mathbf{v}\right|_{T_{i}} \in P_{0}^{2}\left(T_{i}\right)+x \cdot P_{0}\left(T_{i}\right)\right\}, \\
W_{h}=\left\{w \in W: \forall T_{i} \in \mathscr{T}_{h},\left.w\right|_{T_{i}} \in P_{0}\left(T_{i}\right)\right\},
\end{gathered}
$$

where $P_{0}\left(T_{i}\right)$ indicates a constant on $T_{i}$. To approximate the control, we use the following cone of nonnegative piecewise constant functions:

$$
U_{h}=\left\{\tilde{u} \in U_{\text {ad }}:\left.\tilde{u}\right|_{T_{i}}=\text { constant, } \quad \forall T_{i} \in \mathscr{T}_{h}\right\} .
$$

Then we introduce the following Raviart-Thomas projection ${ }^{16}$ :

$$
\Pi_{h} \times P_{h}: \mathbf{V} \times W \longrightarrow \mathbf{V}_{h} \times W_{h},
$$

which has the following properties.

(i) $P_{h}$ is the local $L^{2}(\Omega)$ projection.

(ii) $\Pi_{h}$ and $P_{h}$ satisfy

$$
\operatorname{div} \circ \Pi_{h}=P_{h} \circ \operatorname{div} .
$$

Using property (i) and (13), we can obtain

$$
\begin{gathered}
\left(\operatorname{div}\left(\mathbf{v}-\Pi_{h} \mathbf{v}\right), w_{h}\right)=0, w_{h} \in W_{h}, \\
\left(\operatorname{div} \mathbf{v}_{h}, w-P_{h} w\right)=0, \mathbf{v}_{h} \in \mathbf{V}_{h} .
\end{gathered}
$$


(iii) The following approximate properties hold ${ }^{17}$ :

$$
\begin{gathered}
\left\|\mathbf{v}-\Pi_{h} \mathbf{v}\right\|_{0, \rho} \leqslant C h^{r}\|\mathbf{v}\|_{r, \rho}, \frac{1}{\rho}<r \leqslant k+1 \\
\left\|\operatorname{div}\left(\mathbf{v}-\Pi_{h} \mathbf{v}\right)\right\|_{-t} \leqslant C h^{r+t}\|\operatorname{div} \mathbf{v}\|_{r}, \\
0 \leqslant r, t \leqslant k+1 \\
\left\|w-P_{h} w\right\|_{-t, \rho} \leqslant C h^{r+t}\|w\|_{r, \rho} \\
0 \leqslant r, t \leqslant k+1
\end{gathered}
$$

where $\|.\|_{r, \rho}$ denotes the norm of the usual Sobolev space $W^{r, \rho}(\Omega)$ for $1 \leqslant \rho \leqslant+\infty$ and $r \geqslant 0$.

The mixed finite element approximation of (5)-(7) is to find $\left(\mathbf{p}_{h}, y_{h}, u_{h}\right) \in \mathbf{V}_{h} \times W_{h} \times U_{h}$ such that

$$
\begin{gathered}
\min _{u \in U_{h}} \frac{1}{2}\left\{\left\|\mathbf{p}_{h}-\mathbf{p}_{\mathrm{d}}\right\|^{2}+\left\|y_{h}-y_{\mathrm{d}}\right\|^{2}+\alpha\left\|u_{h}\right\|^{2}\right\} \\
\left(A^{-1} \mathbf{p}_{h}, \mathbf{v}_{h}\right)-\left(y_{h}, \operatorname{div} \mathbf{v}_{h}\right)=0, \\
\left(\operatorname{div} \mathbf{p}_{h}, w_{h}\right)+\left(\phi\left(y_{h}\right), w_{h}\right)=\left(u_{h}, w_{h}\right),
\end{gathered}
$$

where $\mathbf{v}_{h} \in \mathbf{V}_{h}$ and $w_{h} \in W_{h}$.

The optimal control problem (19)-(21) again has a solution $\left(\mathbf{p}_{h}^{*}, y_{h}^{*}, u_{h}^{*}\right)$, and that if a triplet $\left(\mathbf{p}_{h}^{*}, y_{h}^{*}, u_{h}^{*}\right) \in \mathbf{V}_{h} \times W_{h} \times U_{h}$ is the solution of (19)(21), then there is a co-state $\left(\mathbf{q}_{h}^{*}, z_{h}^{*}\right) \in \mathbf{V}_{h} \times W_{h}$ such that $\left(\mathbf{p}_{h}^{*}, y_{h}^{*}, \mathbf{q}_{h}^{*}, z_{h}^{*}, u_{h}^{*}\right)$ satisfies the following discretized optimality conditions:

$$
\begin{gathered}
\left(A^{-1} \mathbf{p}_{h}^{*}, \mathbf{v}_{h}\right)-\left(y_{h}^{*}, \operatorname{div} \mathbf{v}_{h}\right)=0 \\
\left(\operatorname{div} \mathbf{p}_{h}^{*}, w_{h}\right)+\left(\phi\left(y_{h}^{*}\right), w_{h}\right)=\left(u_{h}^{*}, w_{h}\right), \\
\left(A^{-1} \mathbf{q}_{h}^{*}, \mathbf{v}_{h}\right)-\left(z_{h}^{*}, \operatorname{div} \mathbf{v}_{h}\right)=-\left(\mathbf{p}_{h}^{*}-\mathbf{p}_{\mathrm{d}}, \mathbf{v}_{h}\right), \\
\left(\operatorname{div} \mathbf{q}_{h}^{*}, w_{h}\right)+\left(\phi^{\prime}\left(y_{h}^{*}\right) z_{h}^{*}, w_{h}\right)=\left(y_{h}^{*}-y_{\mathrm{d}}, w_{h}\right), \\
\left(z_{h}^{*}+\alpha u_{h}^{*}, \tilde{u}_{h}-u_{h}^{*}\right) \geqslant 0
\end{gathered}
$$

where $\mathbf{v}_{h} \in \mathbf{V}_{h}, w_{h} \in W_{h}$ and $\tilde{u}_{h} \in U_{h}$.

We now shall use some intermediate variables. For any control function $\tilde{u} \in U_{\text {ad }}$, we define the state solution ( $\left.\mathbf{p}^{*}(\tilde{u}), y^{*}(\tilde{u}), \mathbf{q}^{*}(\tilde{u}), z^{*}(\tilde{u})\right)$ associated with $\tilde{u}$ which satisfies

$$
\begin{gathered}
\left(A^{-1} \mathbf{p}^{*}(\tilde{u}), \mathbf{v}\right)-\left(y^{*}(\tilde{u}), \operatorname{div} \mathbf{v}\right)=0, \\
\left(\operatorname{div} \mathbf{p}^{*}(\tilde{u}), w\right)+\left(\phi\left(y^{*}(\tilde{u})\right), w\right)=(\tilde{u}, w), \\
\left(A^{-1} \mathbf{q}^{*}(\tilde{u}), \mathbf{v}\right)-\left(z^{*}(\tilde{u}), \operatorname{div} \mathbf{v}\right) \\
=-\left(\mathbf{p}^{*}(\tilde{u})-\mathbf{p}_{\mathrm{d}}, \mathbf{v}\right), \\
\left(\operatorname{div} \mathbf{q}^{*}(\tilde{u}), w\right)+\left(\phi^{\prime}\left(y^{*}(\tilde{u})\right) z^{*}(\tilde{u}), w\right) \\
=\left(y^{*}(\tilde{u})-y_{\mathrm{d}}, w\right),
\end{gathered}
$$

where $\mathbf{v} \in \mathbf{V}$ and $w \in W$. We define the discrete state solution $\left(\mathbf{p}_{h}^{*}(\tilde{u}), y_{h}^{*}(\tilde{u}), \mathbf{q}_{h}^{*}(\tilde{u}), z_{h}^{*}(\tilde{u})\right)$ corresponding to $\tilde{u}$ which satisfies

$$
\begin{gathered}
\left(A^{-1} \mathbf{p}_{h}^{*}(\tilde{u}), \mathbf{v}_{h}\right)-\left(y_{h}^{*}(\tilde{u}), \operatorname{div} \mathbf{v}_{h}\right)=0, \\
\left(\operatorname{div} \mathbf{p}_{h}^{*}(\tilde{u}), w_{h}\right)+\left(\phi\left(y_{h}^{*}(\tilde{u})\right), w_{h}\right)=\left(\tilde{u}, w_{h}\right), \\
\left(A^{-1} \mathbf{q}_{h}^{*}(\tilde{u}), \mathbf{v}_{h}\right)-\left(z_{h}^{*}(\tilde{u}), \operatorname{div} \mathbf{v}_{h}\right) \\
=-\left(\mathbf{p}_{h}^{*}(\tilde{u})-\mathbf{p}_{\mathrm{d}}, \mathbf{v}_{h}\right), \\
\left(\operatorname{div} \mathbf{q}_{h}^{*}(\tilde{u}), w_{h}\right)+\left(\phi^{\prime}\left(y_{h}^{*}(\tilde{u})\right) z_{h}^{*}(\tilde{u}), w_{h}\right) \\
=\left(y_{h}^{*}(\tilde{u})-y_{\mathrm{d}}, w_{h}\right),
\end{gathered}
$$

where $\mathbf{v}_{h} \in \mathbf{V}_{h}$ and $w_{h} \in W_{h}$. With these definitions, the exact state solution and its approximation can be written as

$$
\begin{aligned}
& \left(\mathbf{p}^{*}, y^{*}, \mathbf{q}^{*}, z^{*}\right)=\left(\mathbf{p}^{*}\left(u^{*}\right), y^{*}\left(u^{*}\right), \mathbf{q}^{*}\left(u^{*}\right), z^{*}\left(u^{*}\right)\right), \\
& \left(\mathbf{p}_{h}^{*}, y_{h}^{*}, \mathbf{q}_{h}^{*}, z_{h}^{*}\right)=\left(\mathbf{p}_{h}^{*}\left(u_{h}^{*}\right), y_{h}^{*}\left(u_{h}^{*}\right), \mathbf{q}_{h}^{*}\left(u_{h}^{*}\right), z_{h}^{*}\left(u_{h}^{*}\right)\right) .
\end{aligned}
$$

For $\varphi \in W_{h}$, we shall write ${ }^{18}$

$$
\begin{aligned}
& \phi(\varphi)-\phi(\rho)=-\tilde{\phi}^{\prime}(\varphi)(\rho-\varphi) \\
& \quad=-\phi^{\prime}(\rho)(\rho-\varphi)+\tilde{\phi}^{\prime \prime}(\varphi)(\rho-\varphi)^{2},
\end{aligned}
$$

where $\tilde{\phi}^{\prime}(\varphi)=\int_{0}^{1} \phi^{\prime}(\varphi+t(\rho-\varphi)) \mathrm{d} t, \tilde{\phi}^{\prime \prime}(\varphi)=$ $\int_{0}^{1}(1-t) \phi^{\prime \prime}(\rho+t(\varphi-\rho)) \mathrm{d} t$ are bounded functions in $\bar{\Omega}$.

\section{SUPERCONVERGENCE}

Firstly, we can obtain the following technical results ${ }^{19}$ :

Lemma 2 Suppose (A1) hold. Let $\gamma \in C^{1}(\Omega), \omega \in \mathbf{V}$, $\varphi \in L^{2}(\Omega)^{2}$, and $\psi \in L^{2}(\Omega)$. If $\tau \in W_{h}$ satisfies

$$
\begin{aligned}
& \left(A^{-1} \omega, \mathbf{v}_{h}\right)-\left(\tau, \operatorname{div} \mathbf{v}_{h}\right)=\left(\varphi, \mathbf{v}_{h}\right), \quad \forall \mathbf{v}_{h} \in \mathbf{V}_{h}, \\
& \left(\operatorname{div} \omega, w_{h}\right)+\left(\gamma \tau, w_{h}\right)=\left(\psi, w_{h}\right), \quad \forall w_{h} \in W_{h},
\end{aligned}
$$

then there exists a constant $C$ such that

$$
\|\tau\|_{0} \leqslant C\left(h\|\omega\|_{0}+h^{2}\|\operatorname{div} \omega\|_{0}+\|\varphi\|_{0}+\|\psi\|_{0}\right),
$$

for h sufficiently small.

For any $\tilde{u} \in U$, let

$$
\varepsilon_{1}:=\mathbf{p}^{*}(\tilde{u})-\mathbf{p}_{h}^{*}(\tilde{u}), \quad e_{1}:=y^{*}(\tilde{u})-y_{h}^{*}(\tilde{u}) .
$$

To analyse the intermediate errors, let us first note the following error equations from (22)-(23) and (27)-(28):

$$
\begin{gathered}
\left(A^{-1} \varepsilon_{1}, \mathbf{v}_{h}\right)-\left(e_{1}, \operatorname{div} \mathbf{v}_{h}\right)=0, \\
\left(\operatorname{div} \varepsilon_{1}, w_{h}\right)+\left(\tilde{\phi}^{\prime}\left(y^{*}(\tilde{u})\right) e_{1}, w_{h}\right)=0,
\end{gathered}
$$

where $\mathbf{v}_{h} \in \mathbf{V}_{h}$ and $w_{h} \in W_{h}$.

By using Lemma 2, we can establish the following error estimates: 
Lemma 3 Suppose that assumptions (A1-3) are fulfilled. Let $y^{*}(\tilde{u})$ and $y_{h}^{*}(\tilde{u})$ be the solutions of (27)(30) and (31)-(34), respectively. If the intermediate solutions satisfy $\mathbf{p}^{*}(\tilde{u}) \in\left[H^{2}(\Omega)\right]^{2}, y^{*}(\tilde{u}) \in H^{4}(\Omega)$ then we have

$$
\left\|y_{h}^{*}(\tilde{u})-y^{*}(\tilde{u})\right\|+\left\|\mathbf{p}_{h}^{*}(\tilde{u})-\mathbf{p}^{*}(\tilde{u})\right\|_{\text {div }} \leqslant C h^{2} .
$$

Lemma 4 Suppose that assumptions (A1-3) are fulfilled. Let $z^{*}(\tilde{u})$ and $z_{h}^{*}(\tilde{u})$ be the solutions of (27)-(30) and (31)-(34), respectively. If the intermediate solutions satisfy $\mathbf{p}^{*}(\tilde{u}), \mathbf{q}^{*}(\tilde{u}) \in\left[H^{2}(\Omega)\right]^{2}$, $y^{*}(\tilde{u}), z^{*}(\tilde{u}) \in H^{4}(\Omega)$ then

$$
\left\|z_{h}^{*}(\tilde{u})-z^{*}(\tilde{u})\right\|+\left\|\mathbf{q}_{h}^{*}(\tilde{u})-\mathbf{q}^{*}(\tilde{u})\right\|_{\text {div }} \leqslant C h^{2} .
$$

Lemma 5 Suppose that assumptions (A1-3) are valid. Let $P_{h} u^{*}$ be the local $L^{2}(\Omega)$ projection of the exact control $u^{*}$ and $z^{*}\left(P_{h} u^{*}\right)$ and $z^{*}\left(u^{*}\right)$ be the solutions of (27)-(30) with $\tilde{u}=P_{h} u^{*}$ and $\tilde{u}=u^{*}$, respectively. Then we have

$$
\left\|z^{*}\left(P_{h} u^{*}\right)-z^{*}\left(u^{*}\right)\right\| \leqslant C h^{2} .
$$

Let $\left(\mathbf{p}^{*}\left(u^{*}\right), y^{*}\left(u^{*}\right)\right)$ and $\left(\mathbf{p}_{h}^{*}\left(u_{h}^{*}\right), y_{h}^{*}\left(u_{h}^{*}\right)\right)$ be the solutions of (8)-(12) and (22)-(26), respectively. Let $J(\cdot): U \rightarrow \mathbb{R}$ be a $G$-differential convex functional with the following form:

$$
J\left(u^{*}\right)=\frac{1}{2}\left\|\mathbf{p}^{*}-\mathbf{p}_{\mathrm{d}}\right\|^{2}+\frac{1}{2}\left\|y^{*}-y_{\mathrm{d}}\right\|^{2}+\frac{\alpha}{2}\left\|u^{*}\right\|^{2} .
$$

It can be shown that

$$
\begin{aligned}
& \left(J^{\prime}\left(u^{*}\right), v\right)=\left(z^{*}+\alpha u^{*}, v\right), \\
& \left(J^{\prime}\left(u_{h}^{*}\right), v\right)=\left(z^{*}\left(u_{h}^{*}\right)+\alpha u_{h}^{*}, v\right) .
\end{aligned}
$$

In many applications, $J(\cdot)$ is uniform convex near the solution $u^{*}$ (see Ref. 5). Then there is a $c>0$, independent of $h$, such that

$$
\left(J^{\prime}\left(u^{*}\right)-J^{\prime}\left(u_{h}^{*}\right), u^{*}-u_{h}^{*}\right) \geqslant c\left\|u^{*}-u_{h}^{*}\right\|^{2},
$$

where $u^{*}$ and $u_{h}^{*}$ are the solutions of (12) and (26), respectively. The convexity of $J(\cdot)$ is closely related to the second order sufficient conditions of the optimal control problem, which are assumed in many studies on numerical methods of the problem.

Let

$$
\begin{aligned}
& \Omega^{+}=\left\{\cup T_{i}: T_{i} \in \Omega,\left.u^{*}\right|_{T_{i}}>0\right\}, \\
& \Omega^{0}=\left\{\cup T_{i}: T_{i} \in \Omega,\left.u^{*}\right|_{T_{i}}=0\right\}, \\
& \Omega^{\mathrm{b}}=\Omega \backslash\left(\Omega^{+} \cup \Omega^{0}\right) .
\end{aligned}
$$

We will assume that $u^{*}$ and $\mathscr{T}_{h}$ are regular such that $\left|\Omega^{\mathrm{b}}\right| \leqslant C h$. We are now able to obtain our first main result.
Theorem 1 Suppose that assumptions (A1-3) are satisfied. Let $P_{h} u^{*}$ be the local $L^{2}(\Omega)$ projection of the exact control $u^{*}$ and $u_{h}^{*}$ be the solution of (22)-(26). Then we have the estimate

$$
\left\|P_{h} u^{*}-u_{h}^{*}\right\| \leqslant C h^{3 / 2} .
$$

Proof: Let $\mathbf{v}=u_{h}^{*}$ in (12) and $\mathbf{v}_{h}=P_{h} u^{*}$ in (26). We have

$$
\begin{array}{r}
\left(z^{*}+\alpha u^{*}, u_{h}^{*}-u^{*}\right) \geqslant 0, \\
\left(z_{h}^{*}+\alpha u_{h}^{*}, P_{h} u^{*}-u_{h}^{*}\right) \geqslant 0 .
\end{array}
$$

Adding the two inequalities gives

$$
\begin{aligned}
\left(z_{h}^{*}+\alpha u_{h}^{*}-z^{*}-\alpha u^{*},\right. & \left.P_{h} u^{*}-u_{h}^{*}\right) \\
& +\left(z^{*}+\alpha u^{*}, P_{h} u^{*}-u^{*}\right) \geqslant 0 .
\end{aligned}
$$

So we obtain

$$
\begin{aligned}
& \alpha\left(P_{h} u^{*}-u_{h}^{*}, P_{h} u^{*}-u_{h}^{*}\right) \\
& =\alpha\left(u^{*}-u_{h}^{*}, P_{h} u^{*}-u_{h}^{*}\right) \\
& \leqslant\left(z_{h}^{*}-z^{*}, P_{h} u^{*}-u_{h}^{*}\right) \\
& \quad+\left(z^{*}+\alpha u^{*}, P_{h} u^{*}-u^{*}\right) .
\end{aligned}
$$

Clearly,

$$
\begin{gathered}
\left(z_{h}^{*}-z^{*}, P_{h} u^{*}-u_{h}^{*}\right)=\left(z_{h}^{*}-z^{*}\left(u_{h}^{*}\right), P_{h} u^{*}-u_{h}^{*}\right) \\
+\left(z^{*}\left(u_{h}^{*}\right)-z^{*}\left(P_{h} u^{*}\right), P_{h} u^{*}-u_{h}^{*}\right) \\
+\left(z^{*}\left(P_{h} u^{*}\right)-z^{*}\left(u^{*}\right), P_{h} u^{*}-u_{h}^{*}\right) .
\end{gathered}
$$

Then

$$
\begin{aligned}
& \alpha\left(P_{h} u^{*}-u_{h}^{*}, P_{h} u^{*}-u_{h}^{*}\right) \\
& \quad-\left(z^{*}\left(u_{h}^{*}\right)-z^{*}\left(P_{h} u^{*}\right), P_{h} u^{*}-u_{h}^{*}\right) \\
& \leqslant\left(z_{h}^{*}-z^{*}\left(u_{h}^{*}\right), P_{h} u^{*}-u_{h}^{*}\right) \\
& \quad+\left(z^{*}\left(P_{h} u^{*}\right)-z^{*}\left(u^{*}\right), P_{h} u^{*}-u_{h}^{*}\right) \\
& \quad+\left(z^{*}+\alpha u^{*}, P_{h} u^{*}-u^{*}\right) \\
& \equiv E_{1}+E_{2}+E_{3} .
\end{aligned}
$$

Now we find bounds for the $E_{i}$. From Lemma 4, we have

$$
\begin{aligned}
E_{1} & =\left(z_{h}^{*}-z^{*}\left(u_{h}^{*}\right), P_{h} u^{*}-u_{h}^{*}\right) \\
& \leqslant C\left\|z_{h}^{*}-z^{*}\left(u_{h}^{*}\right)\right\| \cdot\left\|P_{h} u^{*}-u_{h}^{*}\right\| \\
& \leqslant C h^{2}\left\|P_{h} u^{*}-u_{h}^{*}\right\| .
\end{aligned}
$$

From (40),

$$
\begin{aligned}
E_{2} & =\left(z^{*}\left(P_{h} u^{*}\right)-z^{*}\left(u^{*}\right), P_{h} u^{*}-u_{h}^{*}\right) \\
& \leqslant C\left\|z^{*}\left(P_{h} u^{*}\right)-z^{*}\left(u^{*}\right)\right\| \cdot\left\|P_{h} u^{*}-u_{h}^{*}\right\| \\
& \leqslant C h^{2}\left\|P_{h} u^{*}-u_{h}^{*}\right\| .
\end{aligned}
$$


Finally,

$$
\begin{aligned}
E_{3}=\left(z^{*}+\alpha u^{*}, P_{h} u^{*}-u^{*}\right) & \\
= & \int_{\Omega^{+}}\left(z^{*}+\alpha u^{*}, P_{h} u^{*}-u^{*}\right) \mathrm{d} x \\
& +\int_{\Omega^{0}}\left(z^{*}+\alpha u^{*}, P_{h} u^{*}-u^{*}\right) \mathrm{d} x \\
& +\int_{\Omega^{\mathrm{b}}}\left(z^{*}+\alpha u^{*}, P_{h} u^{*}-u^{*}\right) \mathrm{d} x .
\end{aligned}
$$

From the definition of $\Omega^{0}$ we note that $\left(P_{h} u^{*}-\right.$ $\left.u^{*}\right)\left.\right|_{\Omega^{0}}=0$. It is clear that

$$
\int_{\Omega^{0}}\left(z^{*}+\alpha u^{*}, P_{h} u^{*}-u^{*}\right) \mathrm{d} x=0 .
$$

From (12), we have pointwise a.e. $z^{*}+\alpha u^{*} \geqslant$ 0 . We choose $\left.\tilde{u}\right|_{\Omega^{+}}=0$ and $\left.\tilde{u}\right|_{\Omega \backslash \Omega^{+}}=u^{*}$ so that $\left.\left(z^{*}+\alpha u^{*}, u^{*}\right)\right|_{\Omega^{+}} \leqslant 0$. Hence $\left.\left(z^{*}+\alpha u^{*}\right)\right|_{\Omega^{+}}=0$. Let $\pi^{c} u^{*}$ be the integral operator such that $\left.\pi^{c} u^{*}\right|_{T_{i}}=$ $\int_{T_{i}} u^{*} / \int_{T_{i}} 1$. It follows from the definition of $\pi^{c}$ that

$$
\begin{aligned}
\left(z^{*}\right. & \left.+\alpha u^{*}, P_{h} u^{*}-u^{*}\right) \\
& =\left(z^{*}+\alpha u^{*}, P_{h} u^{*}-u^{*}\right)_{\Omega^{\mathrm{b}}} \\
& \leqslant\left(z^{*}+\alpha u^{*}-\pi^{c}\left(z^{*}+\alpha u^{*}\right), P_{h} u^{*}-u^{*}\right)_{\Omega^{\mathrm{b}}} \\
& \leqslant C h^{2}\left\|z^{*}+\alpha u^{*}\right\|_{1, \Omega^{\mathrm{b}}}\left\|u^{*}\right\|_{1, \Omega^{\mathrm{b}}} \\
& \leqslant C h^{2}\left\|z^{*}+\alpha u^{*}\right\|_{1, \infty}\left\|u^{*}\right\|_{1, \infty} \cdot\left|\Omega^{\mathrm{b}}\right| \\
& \leqslant C h^{3} .
\end{aligned}
$$

Then it follows from assumption (41), (45)-(50), and the Schwartz inequality that

$$
\begin{aligned}
& c\left\|P_{h} u^{*}-u^{*}\right\|_{0}^{2} \\
& \leqslant\left(J^{\prime}\left(P_{h} u^{*}\right)-J^{\prime}\left(u_{h}^{*}\right), P_{h} u^{*}-u^{*}\right) \\
& =\alpha\left(P_{h} u^{*}-u_{h}^{*}, P_{h} u^{*}-u_{h}^{*}\right) \\
& \quad \quad-\left(z^{*}\left(u_{h}^{*}\right)-z^{*}\left(P_{h} u^{*}\right), P_{h} u^{*}-u_{h}^{*}\right) \\
& \leqslant C h^{3}+C h^{2}\left\|P_{h} u^{*}-u_{h}^{*}\right\| \\
& \leqslant C h^{3}+\delta\left\|P_{h} u^{*}-u_{h}^{*}\right\|^{2} .
\end{aligned}
$$

The estimate (42) follows from taking $\delta=\frac{1}{2} c$.

Next, we establish the following superconvergence result for state and co-state.

Theorem 2 Suppose that assumptions (A1-3) are satisfied. Let $\left(\mathbf{p}^{*}, y^{*}, \mathbf{q}^{*}, z^{*}, u^{*}\right) \in(\mathbf{V} \times W)^{2} \times U_{\text {ad }}$ be the solutions defined in (8)-(12) and $\left(\mathbf{p}_{h}^{*}, y_{h}^{*}, \mathbf{q}_{h}^{*}, z_{h}^{*}, u_{h}^{*}\right) \in$ $\left(\mathbf{V}_{h} \times W_{h}\right)^{2} \times U_{h}$ be the solutions of $(22)-(26)$. Then we have

$$
\begin{gathered}
\left\|\Pi_{h} \mathbf{p}^{*}-\mathbf{p}_{h}^{*}\right\|_{\text {div }}+\left\|P_{h} y^{*}-y_{h}^{*}\right\| \leqslant C h^{3 / 2} \\
\left\|\Pi_{h} \mathbf{q}^{*}-\mathbf{q}_{h}^{*}\right\|_{\text {div }}+\left\|P_{h} z^{*}-z_{h}^{*}\right\| \leqslant C h^{3 / 2}
\end{gathered}
$$

Proof: It follows from (8)-(12) and (22)-(26) that we have the error equations:

$$
\begin{aligned}
& \left(A^{-1}\left(\mathbf{p}^{*}-\mathbf{p}_{h}^{*}\right), \mathbf{v}_{h}\right)-\left(y^{*}-y_{h}^{*}, \operatorname{div} \mathbf{v}_{h}\right)=0, \\
& \left(\operatorname{div}\left(\mathbf{p}^{*}-\mathbf{p}_{h}^{*}\right), w_{h}\right)+\left(\tilde{\phi}^{\prime}\left(y^{*}\right)\left(y^{*}-y_{h}^{*}\right), w_{h}\right) \\
& \quad=\left(u^{*}-u_{h}^{*}, w_{h}\right), \\
& \left(A^{-1}\left(\mathbf{q}^{*}-\mathbf{q}_{h}^{*}\right), \mathbf{v}_{h}\right)-\left(z^{*}-z_{h}^{*}, \operatorname{div} \mathbf{v}_{h}\right) \\
& \quad=-\left(\mathbf{p}^{*}-\mathbf{p}_{h}^{*}, \mathbf{v}_{h}\right) \\
& \left(\operatorname{div}\left(\mathbf{q}^{*}-\mathbf{q}_{h}^{*}\right), w_{h}\right)+\left(\phi^{\prime}\left(y^{*}\right)\left(z^{*}-z_{h}^{*}\right), w_{h}\right) \\
& \quad=\left(y^{*}-y_{h}^{*}, w_{h}\right)-\left(\tilde{\phi}^{\prime \prime}\left(y^{*}\right)\left(y^{*}-y_{h}^{*}\right) z_{h}^{*}, w_{h}\right),
\end{aligned}
$$

for all $\mathbf{v}_{h} \in \mathbf{V}_{h}$ and $w_{h} \in W_{h}$. By using the definitions of projections $\Pi_{h}$ and $P_{h}$, the above equations can be rewritten as

$$
\begin{aligned}
& \left(A^{-1}\left(\Pi_{h} \mathbf{p}^{*}-\mathbf{p}_{h}^{*}\right), \mathbf{v}_{h}\right)-\left(P_{h} y^{*}-y_{h}^{*}, \operatorname{div} \mathbf{v}_{h}\right) \\
& \quad=\phi_{1}\left(\mathbf{v}_{h}\right), \\
& \left(\operatorname{div}\left(\Pi_{h} \mathbf{p}^{*}-\mathbf{p}_{h}^{*}\right), w_{h}\right) \\
& \quad+\left(\tilde{\phi}^{\prime}\left(y^{*}\right)\left(P_{h} y^{*}-y_{h}^{*}\right), w_{h}\right)=\psi_{1}\left(w_{h}\right), \\
& \left(A^{-1}\left(\Pi_{h} \mathbf{q}^{*}-\mathbf{q}_{h}^{*}\right), \mathbf{v}_{h}\right) \\
& \quad-\left(P_{h} z^{*}-z_{h}^{*}, \operatorname{div} \mathbf{v}_{h}\right)=\phi_{2}\left(\mathbf{v}_{h}\right), \\
& \left(\operatorname{div}\left(\Pi_{h} \mathbf{q}^{*}-\mathbf{q}_{h}^{*}\right), w_{h}\right) \\
& \quad+\left(\phi^{\prime}\left(y^{*}\right)\left(P_{h} z^{*}-z_{h}^{*}\right), w_{h}\right)=\psi_{2}\left(w_{h}\right),
\end{aligned}
$$

for all $\mathbf{v}_{h} \in \mathbf{V}_{h}$ and $w_{h} \in W_{h}$, where

$$
\begin{aligned}
\phi_{1}\left(\mathbf{v}_{h}\right)=- & \left(A^{-1}\left(\mathbf{p}^{*}-\Pi_{h} \mathbf{p}^{*}\right), \mathbf{v}_{h}\right), \\
\psi_{1}\left(w_{h}\right)= & \left(u^{*}-u_{h}^{*}, w_{h}\right)-\left(\tilde{\phi}^{\prime}\left(y^{*}\right)\left(y^{*}-P_{h} y^{*}\right), w_{h}\right), \\
\phi_{2}\left(\mathbf{v}_{h}\right)=- & \left(\mathbf{p}^{*}-\mathbf{p}_{h}^{*}, \mathbf{v}_{h}\right)-\left(A^{-1}\left(\mathbf{q}^{*}-\Pi_{h} \mathbf{q}^{*}\right), \mathbf{v}_{h}\right), \\
\psi_{2}\left(w_{h}\right)=\left(y^{*}-y_{h}^{*}, w_{h}\right)-\left(\phi^{\prime}\left(y^{*}\right)\left(z^{*}-P_{h} z^{*}\right), w_{h}\right) & -\left(\tilde{\phi}^{\prime \prime}\left(y^{*}\right)\left(y^{*}-y_{h}^{*}\right) z_{h}^{*}, w_{h}\right) .
\end{aligned}
$$

since the terms $\phi_{1}\left(\mathbf{v}_{h}\right), \psi_{1}\left(w_{h}\right), \phi_{2}\left(\mathbf{v}_{h}\right), \psi_{2}\left(w_{h}\right)$ can be regarded as linear functionals of $\mathbf{v}_{h}$ and $w_{h}$ defined on $\mathbf{V}_{h}$ and $W_{h}$, respectively. Then we know from the stability result ${ }^{19,20}$ that

$$
\begin{aligned}
& \left\|\Pi_{h} \mathbf{p}^{*}-\mathbf{p}_{h}^{*}\right\|_{\text {div }}+\left\|P_{h} y^{*}-y_{h}^{*}\right\| \\
& \quad \leqslant C\left\{\sup _{\mathbf{v}_{h} \in \mathbf{V}_{h}} \frac{\left|\phi_{1}\left(\mathbf{v}_{h}\right)\right|}{\left\|\mathbf{v}_{h}\right\|_{\text {div }}}+\sup _{w_{h} \in W_{h}} \frac{\left|\psi_{1}\left(w_{h}\right)\right|}{\left\|w_{h}\right\|}\right\}, \\
& \left\|\Pi_{h} \mathbf{q}^{*}-\mathbf{q}_{h}^{*}\right\|_{\text {div }}+\left\|P_{h} z^{*}-z_{h}^{*}\right\| \\
& \quad \leqslant C\left\{\sup _{\mathbf{v}_{h} \in \mathbf{V}_{h}} \frac{\left|\phi_{2}\left(\mathbf{v}_{h}\right)\right|}{\left\|\mathbf{v}_{h}\right\|_{\text {div }}}+\sup _{w_{h} \in W_{h}} \frac{\left|\psi_{2}\left(w_{h}\right)\right|}{\left\|w_{h}\right\|}\right\} .
\end{aligned}
$$


It is easy to see that

$$
\begin{gathered}
\left(\mathbf{p}^{*}-\mathbf{p}_{h}^{*}, \mathbf{v}_{h}\right)=\left(\mathbf{p}^{*}-\Pi_{h} \mathbf{p}^{*}, \mathbf{v}_{h}\right) \\
+\left(\Pi_{h} \mathbf{p}^{*}-\mathbf{p}_{h}^{*}, \mathbf{v}_{h}\right), \\
\left(y^{*}-y_{h}^{*}, w_{h}\right)=\left(y^{*}-P_{h} y^{*}, w_{h}\right) \\
+\left(P_{h} y^{*}-y_{h}^{*}, w_{h}\right) \\
=\left(P_{h} y^{*}-y_{h}^{*}, w_{h}\right) .
\end{gathered}
$$

By the standard superconvergence of mixed finite element methods ${ }^{21-23}$, we have

$$
\begin{array}{r}
\left(\tilde{\phi}^{\prime}\left(y^{*}\right)\left(y^{*}-P_{h} y^{*}\right), w_{h}\right) \leqslant C h^{2}\left\|y^{*}\right\|_{H^{1}(\Omega)}\left\|w_{h}\right\|, \\
\left(\phi^{\prime}\left(y^{*}\right)\left(z^{*}-P_{h} z^{*}\right), w_{h}\right) \leqslant C h^{2}\left\|z^{*}\right\|_{H^{1}(\Omega)}\left\|w_{h}\right\|, \\
\left(\tilde{\phi}^{\prime \prime}\left(y^{*}\right)\left(y^{*}-P_{h} y^{*}\right) z_{h}^{*}, w_{h}\right) \leqslant C h^{2}\left\|y^{*}\right\|_{H^{1}(\Omega)}\left\|w_{h}\right\| .
\end{array}
$$

Here we only give the proof of (60). By using the definition of the local $L^{2}(\Omega)$ projection $P_{h}$, we obtain

$$
\begin{aligned}
& \left(P_{h}\left(\tilde{\phi}^{\prime}\left(y^{*}\right)\right)\left(y^{*}-P_{h} y^{*}\right), w_{h}\right) \\
& \quad=\left(y^{*}-P_{h} y^{*}, P_{h}\left(\tilde{\phi}^{\prime}\left(y^{*}\right)\right) w_{h}\right)=0 .
\end{aligned}
$$

Then

$$
\begin{aligned}
& \left(\tilde{\phi}^{\prime}\left(y^{*}\right)\left(y^{*}-P_{h} y^{*}\right), w_{h}\right) \\
& \quad=\left(\left(\tilde{\phi}^{\prime}\left(y^{*}\right)-P_{h}\left(\tilde{\phi}^{\prime}\left(y^{*}\right)\right)\right)\left(y^{*}-P_{h} y^{*}\right), w_{h}\right) \\
& \quad \leqslant C h\|\phi\|_{2, \infty}\left\|y^{*}-P_{h} y^{*}\right\|\left\|w_{h}\right\| \\
& \quad \leqslant C h^{2}\left\|y^{*}\right\|_{H^{1}(\Omega)}\left\|w_{h}\right\| .
\end{aligned}
$$

Under the condition $y^{*}, z^{*} \in H^{3}(\Omega)$, applying the integral identity technique ${ }^{24}$, we see that

$$
\begin{aligned}
\left(A^{-1}\left(\mathbf{p}^{*}-\Pi_{h} \mathbf{p}^{*}\right), \mathbf{v}_{h}\right) & \leqslant C h^{2}\left\|y^{*}\right\|_{H^{3}(\Omega)}\left\|\mathbf{v}_{h}\right\|, \\
\left(A^{-1}\left(\mathbf{q}^{*}-\Pi_{h} \mathbf{q}^{*}\right), \mathbf{v}_{h}\right) & \leqslant C h^{2}\left\|z^{*}\right\|_{H^{3}(\Omega)}\left\|\mathbf{v}_{h}\right\|, \\
\left(\mathbf{p}^{*}-\Pi_{h} \mathbf{p}^{*}, \mathbf{v}_{h}\right) & \leqslant C h^{2}\left\|y^{*}\right\|_{H^{3}(\Omega)}\left\|\mathbf{v}_{h}\right\| .
\end{aligned}
$$

By adding (54) and (55) to $\mathbf{v}_{h}=\Pi_{h} \mathbf{p}^{*}-\mathbf{p}_{h}^{*}$ and $w_{h}=P_{h} y^{*}-y_{h}^{*}$, we have

$$
\begin{aligned}
& \left(A^{-1}\left(\Pi_{h} \mathbf{p}^{*}-\mathbf{p}_{h}^{*}\right), \Pi_{h} \mathbf{p}^{*}-\mathbf{p}_{h}^{*}\right) \\
& +\left(\tilde{\phi}^{\prime}\left(y^{*}\right)\left(P_{h} y^{*}-y_{h}^{*}\right), P_{h} y^{*}-y_{h}^{*}\right) \\
& =\left(u^{*}-u_{h}^{*}, P_{h} y^{*}-y_{h}^{*}\right) \\
& -\left(\tilde{\phi}^{\prime}\left(y^{*}\right)\left(y^{*}-P_{h} y^{*}\right), P_{h} y^{*}-y_{h}^{*}\right) \\
& \quad-\left(A^{-1}\left(\mathbf{p}^{*}-\Pi_{h} \mathbf{p}^{*}\right), \Pi_{h} \mathbf{p}^{*}-\mathbf{p}_{h}^{*}\right) .
\end{aligned}
$$

Table 1 The errors of Example 1 on sequential uniform refined meshes.

\begin{tabular}{lll}
\hline resolution & $\left\|u^{*}-u_{h}^{*}\right\|$ & $\left\|P_{h} u^{*}-u_{h}^{*}\right\|$ \\
\hline $16 \times 16$ & $1.133 \times 10^{-1}$ & $2.112 \times 10^{-2}$ \\
$32 \times 32$ & $5.668 \times 10^{-2}$ & $7.168 \times 10^{-3}$ \\
$64 \times 64$ & $2.834 \times 10^{-2}$ & $2.138 \times 10^{-3}$ \\
$128 \times 128$ & $1.417 \times 10^{-2}$ & $6.948 \times 10^{-4}$ \\
\hline
\end{tabular}

Using the assumption of $A(x), \phi$ and Hölder's inequality, for any small $\delta>0$, we obtain

$$
\begin{aligned}
& \frac{1}{c^{*}}\left\|\Pi_{h} \mathbf{p}^{*}-\mathbf{p}_{h}^{*}\right\|^{2}+\lambda\left\|P_{h} y^{*}-y_{h}^{*}\right\|^{2} \\
& \leqslant(\left.A^{-1}\left(\Pi_{h} \mathbf{p}^{*}-\mathbf{p}_{h}^{*}\right), \Pi_{h} \mathbf{p}^{*}-\mathbf{p}_{h}^{*}\right) \\
&+\left(\tilde{\phi}^{\prime}\left(y^{*}\right)\left(P_{h} y^{*}-y_{h}^{*}\right), P_{h} y^{*}-y_{h}^{*}\right) \\
&=\left(u^{*}-u_{h}^{*}, P_{h} y^{*}-y_{h}^{*}\right) \\
&-\left(\tilde{\phi}^{\prime}\left(y^{*}\right)\left(y^{*}-P_{h} y^{*}\right), P_{h} y^{*}-y_{h}^{*}\right) \\
&-\left(A^{-1}\left(\mathbf{p}^{*}-\Pi_{h} \mathbf{p}^{*}\right), \Pi_{h} \mathbf{p}^{*}-\mathbf{p}_{h}^{*}\right) \\
& \leqslant\left\|u^{*}-u_{h}^{*}\right\| \cdot\left\|P_{h} y^{*}-y_{h}^{*}\right\| \\
&+\|\phi\|_{2, \infty}\left\|y^{*}-P_{h} y^{*}\right\| \cdot\left\|P_{h} y^{*}-y_{h}^{*}\right\| \\
&+C\left\|\mathbf{p}^{*}-\Pi_{h} \mathbf{p}^{*}\right\| \cdot\left\|\Pi_{h} \mathbf{p}^{*}-\mathbf{p}_{h}^{*}\right\| \\
& \leqslant\left\|u^{*}-u_{h}^{*}\right\| \cdot\left\|P_{h} y^{*}-y_{h}^{*}\right\| \\
&+C h^{2}\left\|y^{*}\right\|_{H^{2}(\Omega)} \cdot\left\|P_{h} y^{*}-y_{h}^{*}\right\| \\
&+C h^{2}\left\|y^{*}\right\|_{H^{3}(\Omega)} \cdot\left\|\Pi_{h} \mathbf{p}^{*}-\mathbf{p}_{h}^{*}\right\| \\
& \leqslant C h^{4}+C\left\|u^{*}-u_{h}^{*}\right\|^{2} \\
&+\delta\left(\left\|P_{h} y^{*}-y_{h}^{*}\right\|^{2}+\left\|\Pi_{h} \mathbf{p}^{*}-\mathbf{p}_{h}^{*}\right\|^{2}\right) .
\end{aligned}
$$

The formula is equivalent to

$$
\left\|\Pi_{h} \mathbf{p}^{*}-\mathbf{p}_{h}^{*}\right\|+\left\|P_{h} y^{*}-y_{h}^{*}\right\| \leqslant C h^{2}+C\left\|u^{*}-u_{h}^{*}\right\| .
$$

Note that

$$
\left(u^{*}-u_{h}^{*}, w_{h}\right)=\left(u^{*}-P_{h} u^{*}, w_{h}\right)+\left(P_{h} u^{*}-u_{h}^{*}, w_{h}\right) .
$$

It is easy to see that $\left(u^{*}-P_{h} u^{*}, w_{h}\right)=0$. By using Theorem 1 , we clearly see that

$$
\begin{aligned}
\left(P_{h} u^{*}-u_{h}^{*}, w_{h}\right) & \leqslant\left\|P_{h} u^{*}-u_{h}^{*}\right\| \cdot\left\|w_{h}\right\| \\
& \leqslant C h^{3 / 2}\left\|w_{h}\right\| .
\end{aligned}
$$

From the above analysis, we can obtain (52) and (53).

\section{NUMERICAL EXAMPLES}

We present two examples to test the superconvergence results of the control. The optimization problems were solved numerical by projected 


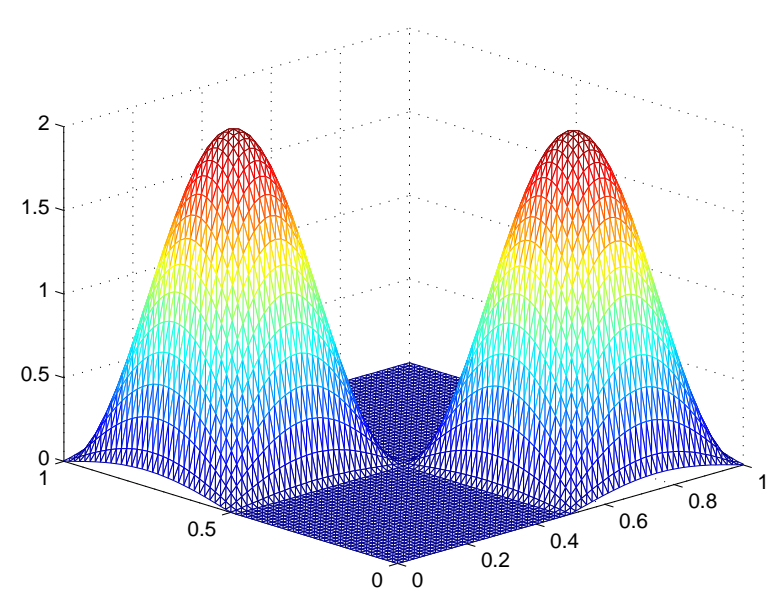

Fig. 1 The profile of the numerical solution of Example 1.

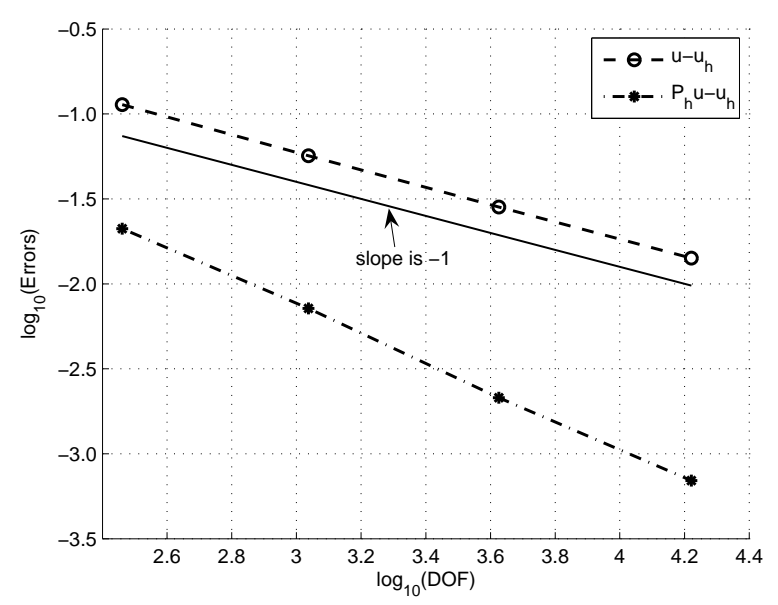

Fig. 2 Convergence orders of $u^{*}-u_{h}^{*}$ and $P_{h} u^{*}-u_{h}^{*}$.

gradient methods, with codes developed based on AFEPACK $^{25}$. The control function $u$ is discretized by piecewise constant functions, where the state $(y, \mathbf{p})$ and the co-state $(z, \mathbf{q})$ were approximated by the lowest order Raviart-Thomas mixed finite element functions. In the two examples, we choose the domain $\Omega=[0,1] \times[0,1]$.

Example 1 We consider the two-dimensional nonlinear elliptic optimal control problem

$$
\min _{u \in U_{\mathrm{ad}}} \frac{1}{2}\left\{\left\|\mathbf{p}-\mathbf{p}_{\mathrm{d}}\right\|^{2}+\left\|y-y_{\mathrm{d}}\right\|^{2}+\|u\|^{2}\right\}
$$

subject to the state equation

$$
\operatorname{div} \mathbf{p}+y^{3}=u+f, \mathbf{p}=-\nabla y, \quad x \in \Omega,
$$

with the boundary condition $y=0, x \in \partial \Omega$, and the admissible set $U_{\text {ad }}=\left\{u \in L^{2}(\Omega): u \geqslant 0\right\}$. Next, we introduce the co-state elliptic equation $\operatorname{div} \mathbf{q}+$
Table 2 The errors of Example 2 on sequential uniform refined meshes.

\begin{tabular}{lcc}
\hline resolution & $\left\|u^{*}-u_{h}^{*}\right\|$ & $\left\|P_{h} u^{*}-u_{h}^{*}\right\|$ \\
\hline $16 \times 16$ & $1.189 \times 10^{-3}$ & $2.621 \times 10^{-4}$ \\
$32 \times 32$ & $5.843 \times 10^{-4}$ & $8.932 \times 10^{-5}$ \\
$64 \times 64$ & $2.891 \times 10^{-4}$ & $3.129 \times 10^{-5}$ \\
$128 \times 128$ & $1.438 \times 10^{-4}$ & $1.109 \times 10^{-5}$ \\
\hline
\end{tabular}

$3 y^{2} z=y-y_{\mathrm{d}}, \mathbf{q}=-\left(\nabla z+\mathbf{p}-\mathbf{p}_{\mathrm{d}}\right), x \in \Omega$, with the boundary condition $z=0, x \in \partial \Omega$. We choose

$$
\begin{aligned}
u & =\max (0,-z), \\
f & =8 \pi^{2} y+y^{3}-u, \\
y_{\mathrm{d}} & =\left(1-16 \pi^{2}\right) y-3 y^{2} z, \\
y & =\sin \left(2 \pi x_{1}\right) \sin \left(2 \pi x_{2}\right), \\
z & =2 \sin \left(2 \pi x_{1}\right) \sin \left(2 \pi x_{2}\right), \\
\mathbf{p} & =-2 \pi\left(\cos 2 \pi x_{1} \sin 2 \pi x_{2}, \cos 2 \pi x_{2} \sin 2 \pi x_{1}\right), \\
\mathbf{q} & =-\pi\left(\cos 2 \pi x_{1} \sin 2 \pi x_{2}, \cos 2 \pi x_{2} \sin 2 \pi x_{1}\right), \\
\mathbf{p}_{\mathrm{d}} & =\pi\left(\cos 2 \pi x_{1} \sin 2 \pi x_{2}, \cos 2 \pi x_{2} \sin 2 \pi x_{1}\right) .
\end{aligned}
$$

In the numerical implementation, the profile of the numerical solution is shown in Fig. 1 and the errors $\left\|u^{*}-u_{h}^{*}\right\|$ and $\left\|P_{h} u^{*}-u_{h}^{*}\right\|$ obtained on a sequence of uniformly refined meshes are presented in Table 1. The convergence orders on triangle mesh grids are depicted in Fig. 2. It is clear that $\left\|P_{h} u^{*}-u_{h}^{*}\right\|$ has a superconvergence of $O\left(h^{3 / 2}\right)$.

Example 2 We consider the following nonlinear optimal control problem:

$$
\begin{aligned}
& \min _{u \in U_{\mathrm{ad}}} \frac{1}{2}\left\{\left\|\mathbf{p}-\mathbf{p}_{\mathrm{d}}\right\|^{2}+\left\|y-y_{\mathrm{d}}\right\|^{2}+\|u\|^{2}\right\}, \\
& \operatorname{div} \mathbf{p}+y^{7}=u+f, \mathbf{p}=-\nabla y, x \in \Omega, \\
& \quad y=0, \quad x \in \partial \Omega,
\end{aligned}
$$

and we introduce co-state elliptic equation $\operatorname{div} \mathbf{q}+$ $7 y^{6} z=y-y_{\mathrm{d}}, \mathbf{q}=-\left(\nabla z+\mathbf{p}-\mathbf{p}_{\mathrm{d}}\right), x \in \Omega$, with the boundary condition $z=0, x \in \partial \Omega$. We choose that

$$
\begin{aligned}
u & =\max (-z, 0), \quad f=\operatorname{div} \mathbf{p}+y^{7}-u, \\
y_{\mathrm{d}} & =y-\operatorname{div} \mathbf{q}-7 y^{6} z, \quad \mathbf{q}=-\nabla z-\mathbf{p}+\mathbf{p}_{\mathrm{d}}, \\
y & =2 x_{1} x_{2}^{2}\left(1-x_{1}^{3}\right)\left(1-x_{2}\right)^{2} \sin \left(8 \pi x_{1}\right), \\
z & =-x_{1} x_{2}^{2}\left(1-x_{1}^{3}\right)\left(1-x_{2}\right)^{2} \sin \left(8 \pi x_{1}\right), \\
\mathbf{p} & =-\nabla y, \quad \mathbf{p}_{\mathrm{d}}=\mathbf{p}+\mathbf{q}+\nabla z
\end{aligned}
$$

The profile of the numerical solution is presented in Fig. 3. The superconvergence behaviour of $\left\|P_{h} u^{*}-u_{h}^{*}\right\|$ is illustrated in Table 2 and Fig. 4. 


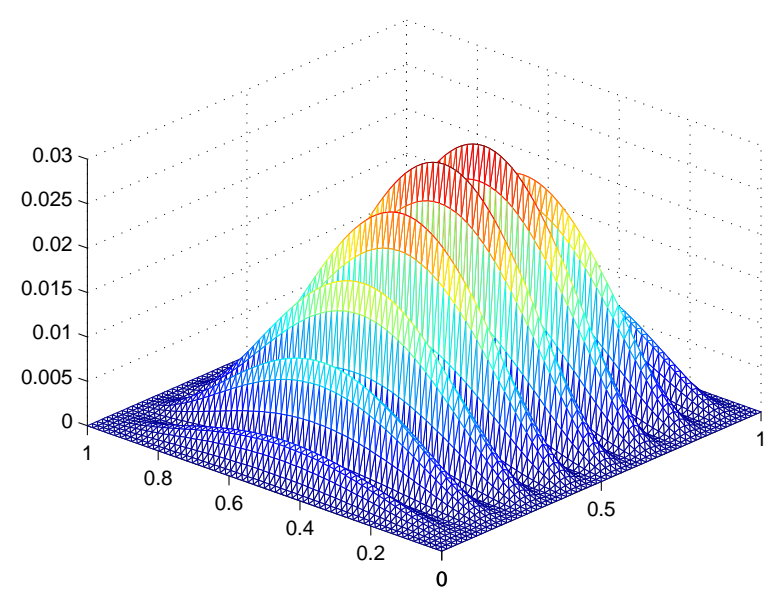

Fig. 3 The profile of the numerical solution of Example 2.

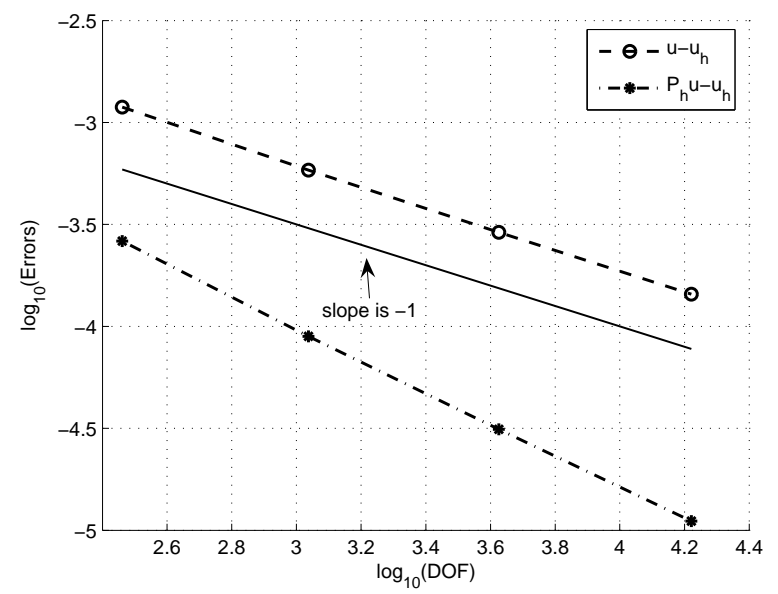

Fig. 4 Convergence orders of $u^{*}-u_{h}^{*}$ and $P_{h} u^{*}-u_{h}^{*}$.

From the numerical results of the examples, the superconvergence phenomenon can be observed clearly.

Acknowledgements: The authors thank the referees for their helpful suggestions, which led to improvements in the presentation. This work was supported by the National Basic Research Programme (2012CB955804), Major Research Plan of National Natural Science Foundation of China (91430108), National Science Foundation of China (11201510, 11171251), China Postdoctoral Science Foundation (2015M580197), Chongqing Research Programme of Basic Research and Frontier Technology (cstc2015jcyjA20001), Ministry of education Chunhui projects (Z2015139), Major Programme of Tianjin University of finance and Economics (ZD1302) and Science and Technology Project of Wanzhou District of Chongqing (2013030050).

\section{REFERENCES}

1. Falk FS (1973) Approximation of a class of optimal control problems with order of convergence estimates. J Math Anal Appl 44, 28-47.

2. Geveci T (1979) On the approximation of the solution of an optimal control problem governed by an elliptic equation. RAIRO Anal Numér 13, 313-28.

3. Arada N, Casas E, Troltzsch F (2002) Error estimates for a semilinear elliptic control problem. Comput Optim Appl 23, 201-29.

4. Meyer C, Rösch A (2004) Superconvergence properties of optimal control problems. SIAM J Contr Optim 43, 970-85.

5. Liu W, Yan N (2001) A posteriori error estimates for distributed convex optimal control problems. $A d v$ Comput Math 15, 285-309.

6. Liu W, Yan N (2001) A posteriori error estimates for control problems governed by nonlinear elliptic equation. Adv Comput Math 15, 285-309.

7. Chen Y, Lu Z (2010) Error estimates for parabolic optimal control problem by fully discrete mixed finite element methods. Finite Elem Anal Des 46, 957-65.

8. Chen Y (2008) Superconvergence of quadratic optimal control problems by triangular mixed finite elements. Int J Numer Meth Eng 75, 881-98.

9. Chen Y, Lu Z (2010) Error estimates of fully discrete mixed finite element methods for semilinear quadratic parabolic optimal control problems. Comput Meth Appl Mech Eng 199, 1415-23.

10. Chen Y (2008) Superconvergence of mixed finite element methods for optimal control problems. Math Comput 77, 1269-91.

11. Chen Y (2008) Superconvergence of quadratic optimal control problems by triangular mixed finite elements. Int J Numer Meth Eng 75, 881-98.

12. Chen Y, Lu Z, Huang Y (2013) Superconvergence of triangular Raviart-Thomas mixed finite element methods for bilinear constrained optimal control problem. Comput Math Appl 66, 1498-513.

13. Bonnans JF (1998) Second-order analysis for control constrained optimal control problems of semilinear elliptic systems. Appl Math Optim 38, 303-25.

14. Chen Y, Lu Z (2015) High Efficient and Accuracy Numerical Methods for Optimal Control Problems, Science Press.

15. Raviart PA, Thomas JM (1977) A mixed finite element method for 2nd order elliptic problems. In: Galligani I (ed) Math Aspects of the Finite Element Method, Springer, Berlin, pp 292-315.

16. Douglas J, Roberts JE (1985) Global estimates for mixed finite element methods for second order elliptic equations. Math Comput 44, 39-52.

17. Lu Z, Chen Y (2009) A posteriori error estimates of triangular mixed finite element methods for semilinear optimal control problems. Adv Appl Math Mech 1, 242-56. 
18. Kwon Y, Milner FA (1988) $L^{\infty}$-error estimates for mixed methods for semilinear second-order elliptic equations. SIAM J Numer Anal 25, 46-53.

19. Milner FA (1985) Mixed finite element methods for quasilinear second-order elliptic problems. Math Comput 44, 303-20.

20. Brezzi F, Fortin M (1991) Mixed and Hybrid Finite Element Methods, Springer, Verlag.

21. Brandts JH (1994) Superconvergence and a posteriori error estimation for triangular mixed finite elements. Numer Math 68, 311-24.

22. Brandts JH (2000) Superconvergence for triangular order $k=1$ Raviart-Thomas mixed finite elements and for triangular standard quadratic finite element methods. Appl Numer Math 34, 39-58.

23. Durán R, Muschietti MA, Rodríguez R (1991) On the asymptotic exactness of error estimators for linear triangular finite elements. Numer Math 59, 107-27.

24. Chen Y, Lu Z, Liu L (2015) Numerical Methods of Partial Differential Equations, Science Press, Beijing.

25. Li R (2005) On multi-mesh $h$-adaptive methods. J Sci Comput 24, 321-41. 